\title{
ARTICLE \\ Impact of sex and depressed mood on the central regulation of cardiac autonomic function
}

\author{
Ronald G. Garcia $\mathbb{D}^{1,2}$, Klara Mareckova ${ }^{3}$, Laura M. Holsen ${ }^{4}$, Justine E. Cohen ${ }^{1}$, Susan Whitfield-Gabrieli ${ }^{5}$, Vitaly Napadow ${ }^{2}$, \\ Riccardo Barbieri ${ }^{6,7}$ and Jill M. Goldstein (iD) ${ }^{1,2,4,8}$
}

\begin{abstract}
Cardiac autonomic dysregulation has been implicated in the comorbidity of major psychiatric disorders and cardiovascular disease, potentially through dysregulation of physiological responses to negative stressful stimuli (here, shortened to stress response). Further, sex differences in these comorbidities are substantial. Here, we tested the hypothesis that mood- and sex-dependent alterations in brain circuitry implicated in the regulation of the stress response are associated with reduced peripheral parasympathetic activity during negative emotional arousal. Fifty subjects ( 28 females) including healthy controls and individuals with major depression, bipolar psychosis and schizophrenia were evaluated. Functional magnetic resonance imaging and physiology (cardiac pulse) data were acquired during a mild visual stress reactivity challenge. Associations between changes in activity and functional connectivity of the stress response circuitry and variations in cardiovagal activity [normalized high frequency power of heart rate variability (HFn)] were evaluated using GLM analyses, including interactions with depressed mood and sex across disorders. Our results revealed that in women with high depressed mood, lower cardiovagal activity in response to negative affective stimuli was associated with greater activation of hypothalamus and right amygdala and reduced connectivity between hypothalamus and right orbitofrontal cortex, amygdala, and hippocampus. No significant associations were observed in women with low levels of depressed mood or men. Our results revealed mood- and sex-dependent interactions in the central regulation of cardiac autonomic activity in response to negative affective stimuli. These findings provide a potential pathophysiological mechanism for previously observed sex differences in the comorbidity of major depression and cardiovascular disease.
\end{abstract}

Neuropsychopharmacology (2020) 45:1280-1288; https://doi.org/10.1038/s41386-020-0651-x

\section{INTRODUCTION}

The dysregulation of neural and physiological responses to negative affective stimuli (subsequently referred to as "stress response circuitry") has been implicated in the pathophysiology of several major psychiatric disorders such as major depression (MDD) and schizophrenia [1-7]. These alterations may be associated with their substantial comorbidity with cardiovascular disease, increasing the risk for the development and progression of coronary artery disease, myocardial infarction and heart failure $[1,7,8]$. We and others have suggested that these maladaptive physiological responses are highly associated with abnormalities in stress response circuitry, which includes brainstem, periaqueductal gray (PAG), hypothalamus (HYPO), amygdala (AMYG), hippocampus (HIPP), anterior cingulate cortex (ACC), medial prefrontal cortex (mPFC), and orbitofrontal cortex (OFC), among others [5, 9-12]. Many of these stress circuitry regions are morphologically and functionally sexually dimorphic beginning in prenatal development [13-18], which we and others have shown predispose for sex differences in the susceptibility for mental disorders and increased risk for cardiovascular disease $[2,7,8,19,20]$. For example, women have twice the risk of men for developing comorbidity of depression and cardiometabolic disorders [21-27] leading to a 3-5-fold risk of death from heart disease in depressed women.

A major role for the stress response circuitry is to coordinate homeostatic responses throughout the body, including modulation of cardiac autonomic and neuroendocrine signals in response to stressful threatening physical or psychological stimuli $[4,5,9]$. Central to this function is the integrative role of the hypothalamus, which receives information from many levels of the central nervous system, including the limbic system, and coordinates peripheral physiological responses through interaction with other subcortical areas $[28,29]$. For instance, in response to stress, the paraventricular nucleus (PVN) of the hypothalamus secretes corticotropin-releasing hormone $(\mathrm{CRH})$, which in turn triggers release of adrenocorticotropin hormone (ACTH) from the anterior pituitary, and subsequently cortisol from the adrenal cortex, regulating metabolism, immune and vascular function [30]. In addition, the hypothalamus via PVN regulates the function of the nucleus tractus solitarii (NTS) and premotor vagal nuclei in the medulla, such as nucleus ambiguus (nAmb) [31, 32], having a direct effect in peripheral parasympathetic activity regulating heart and cardiovascular function.

${ }^{1}$ Department of Psychiatry, Massachusetts General Hospital, Harvard Medical School, Boston, MA, USA; ${ }^{2}$ Martinos Center for Biomedical Imaging, Department of Radiology, Massachusetts General Hospital, Harvard Medical School, Boston, MA, USA; ${ }^{3}$ Brain and Mind Research Programme, Central European Institute of Technology, Masaryk University, Brno, Czech Republic; ${ }^{4}$ Division of Women's Health, Department of Medicine, Brigham and Women's Hospital, Harvard Medical School, Boston, MA, USA; ${ }^{5}$ Department of Psychology, Northeastern University, Boston, MA, USA; ${ }^{6}$ Department of Electronics, Information and Bioengineering, Politecnico di Milano, Milano, Italy; ${ }^{7}$ Department of Anesthesia and Critical Care, Massachusetts General Hospital, Harvard Medical School, Boston, MA, USA and ${ }^{8}$ Department of Obstetrics and Gynecology, Massachusetts General Hospital, Boston, MA, USA

Correspondence: Jill M. Goldstein (jill_goldstein@hms.harvard.edu)

Received: 12 July 2019 Revised: 21 February 2020 Accepted: 28 February 2020

Published online: 9 March 2020 
Multiple studies have demonstrated the presence of HPA axis alterations in MDD patients, such as elevated cortisol levels in plasma, urine and cerebral spinal fluid, as well as blunted responses to $\mathrm{CRH}$ administration, and failure to suppress cortisol secretion following dexamethasone administration [33-38]. Several reports also demonstrated cardiac autonomic dysregulation characterized by decreased parasympathetic activity in MDD and schizophrenia [39-42]. Loss of parasympathetic modulation exposes the heart to unopposed sympathetic excitation, which increases the risk of developing ventricular arrhythmias, sudden death, myocardial infarction and congestive heart failure in patients with MDD [1]. Neuroimaging studies, including ours $[4,6,9,43-45]$, have suggested that dysregulation of HPA axis and autonomic physiology in psychiatric disorders may be caused by alterations in stress response circuitry regions. In particular, studies, including ours, with patients with MDD or psychoses have found hyperactivity of the AMYG, HIPP and HYPO associated with elevated cortisol response to negative stimuli [4, 9, 46, 47]. In addition, we and others have found hypoactivation of ACC and OFC and reduced connectivity of these areas with subcortical areas and brainstem autonomic nuclei correlated with decreased cardiac parasympathetic regulation in depressed individuals $[43,48]$.

Previous findings from our group demonstrated a significant relationship between depressed mood, stress response circuitry alterations and maladaptive HPA axis responses to negative affective stimuli [47]. The current report builds on these previous findings and extends them in the same sample by characterizing the impact of depressive symptomatology on the central neural mechanisms of cardiac autonomic regulation in response to negative affective stimuli. Furthermore, although the incidence of sex differences in the relationship between mood disorders and cardiovascular disease has been known for many years, even recent studies evaluating brain circuitry and physiology associated with mood dysregulation have not been designed to evaluate sexdependent variations. In the study presented here, we evaluated sex differences in stress response circuitry alterations and their associations with autonomic dysregulation, that we believe are shared across psychiatric disorders.

Although neural, behavioral and physiological responses to affective stimuli are usually studied in a dichotomous fashion by comparing healthy populations with patients with a particular psychiatric disorder, they vary on a continuum from adaptive to maladaptive [46, 47]. This offers an opportunity to identify pathophysiological mechanisms or neurobiological signatures associated with behavioral traits rather than diagnoses [49]. We demonstrated previously sex differences in neural function and hormonal physiology associated with response to negative affective stimuli were shared across clinical populations [46, 47]. Here, we extend this work by investigating, in the same population, how brain activity is also associated with peripheral cardiac autonomic physiology in response to negative affective stimuli as a function of sex and depressed mood across disorders. We hypothesized that women with high levels of depressed mood would exhibit hyperactivity of subcortical arousal regions and hypoactivity in cortical regions that would be associated with parasympathetic activity reduction in response to negative affective stimuli. These findings may contribute to elucidating the neural and physiological mechanisms underlying the association between cardiovascular dysfunction and mood dysregulation and the impact of sex.

\section{MATERIALS AND METHODS}

Study population

Fifty adults ( 28 females, 22 males) were included in this analysis. This sample is a subset of the study population evaluated previously by our team [46, 47], and did not differ in sociodemographic characteristics from the larger sample. The majority of the subjects were offspring of women who took part in the large scale $(n=17,741)$ Boston and Providence Collaborative Perinatal Project. This cohort has been followed by our group for over 20 years in the New England Family Studies (NEFS; [2]). Exclusion criteria included contraindications for MRI, the use of caffeine in the morning prior to the fasting blood sample, alcohol consumption $24 \mathrm{~h}$ prior to the blood draw, and any current substance use disorders, as assessed by the Structured Clinical Interview for the DSM (SCID). All participants provided written informed consent to a protocol approved by Harvard University, Brown University, Partners Healthcare system, and local psychiatric facilities and were compensated for their participation.

Assessment of mood and anxiety

The Profile of Mood States (POMS; [50]) and State-Trait Anxiety Inventory (STAl; [51]), self-report questionnaires, were administered to each participant at baseline (before the MRI scan) to assess mood and anxiety symptoms. The POMS questionnaire is composed of five subscales (tension/anxiety, depression/dejection, anger/hostility, fatigue/inertia, vigor/activity, confusion/ bewilderment [52]). For the current analysis we selected the depression/dejection subscale score as a measure of depressed mood across the clinical populations included. We used this measure in regression models evaluating the impact of depressed mood on the relationship between activation and connectivity of the stress response circuitry with cardiac autonomic activity in response to negative affective stimuli. A mean split of the depression/dejection subscale score was used to classify subjects into high or low levels of depressed mood categories.

\section{Steroid hormone assessments}

Cortisol was estimated in response to a negative affect task in real time during functional imaging [4, 9]. An in-scanner blood sample (90 min post-stress challenge) was drawn out-of-scanner in a quiet room. Clotting time was allotted, samples spun to separate sera from blood cells and stored at $-80^{\circ} \mathrm{C}$. The Harvard Clinical Translational Science Center laboratory analyzed samples in duplicate with commercial radioimmunoassay (RIA) kits (cortisol [sensitivity $0.04 \mu \mathrm{g} / \mathrm{dL}$, intra-assay variation $4.4-6.7 \%$ ], Access Immunoassay System, Beckman Coulter, Miami, FL).

\section{Assessment of cardiovagal activity}

A photoplethysmography sensor attached to the right index finger was used during the task to collect cardiac pulse. Data were acquired using the Powerlab system (ML880, ADInstruments Inc., Colorado Springs, CO) at a $1000 \mathrm{~Hz}$ sampling rate. The evaluation of heart rate variability (HRV) provides effective non-invasive measures of cardiac parasympathetic efferent modulation in humans [53-55]. This method typically involves calculating the frequency power spectrum of a continuous estimate of heart rate, and assessing power in the high frequency band (HF, 0.15-0.40 $\mathrm{Hz}$ ), influenced solely by cardiovagal activity [53]. The traditional implementation of this analysis requires quasi-stationarity, which limits its use in tracking rapid changes in temporal dynamics of heartbeat intervals. In this study, a point-process algorithm [56] was applied to accurately quantify cardiovagal control dynamics during the exposure of subjects to negative- or neutral-valence images during the fMRI-visual stress task. Using this method, the time intervals between heart beats were derived from the photoplethismographic pulse signal and corrected for ectopic beats with a previously developed algorithm [57]. The stochastic structure of the inter-beat intervals (IBI) was modeled as an inverse Gaussian renewal process where the current $\mid \mathrm{BI}$ prediction is linearly dependent on the recent history of parasympathetic and sympathetic inputs to the Sinoatrial node, and therefore is a function of the last $p \mid \mathrm{IBI}$, where $p$ is the model order. The HRV point-process model and all its parameters were recursively updated. The model regression order $p$ was chosen using a 
preliminary optimization procedure based on combining Akaike Information Criterion with the Kolmogorov-Smirnov goodness-offit test [58]. Autocorrelation plots were also considered to test the independence of the model-transformed intervals. Once the order $p$ was determined, the initial model coefficients were estimated by the method of least squares [58] for all subjects. The HRV dynamics were then computed in the classic spectral components [low frequency (LF), $0.04-0.15 \mathrm{~Hz}$; high frequency (HF), 0.15-0.4 $\mathrm{Hz}]$, and their time-varying behavior can therefore be updated at any time resolution [58]. The spectral LF component is influenced by both cardiovagal and sympathetic activity, while the HF band is considered to be an index of parasympathetic modulation [53]. In this analysis, a fixed order $p=8$ was chosen and indices were updated every $5 \mathrm{~ms}$. Normalized HF indices were calculated $[\mathrm{HFn}=(\mathrm{HF} /(\mathrm{LF}+\mathrm{HF})]$. HFn differences during exposure to negative compared with neutral images in the fMRI task were estimated and used in GLM analyses. A logarithmic transformation of the difference between HF values during negative and neutral images $\left(\mathrm{LogHF}_{\text {negative-neutral }}\right)$ was also calculated.

\section{Acquisition of $\mathrm{FMRI}$ data}

All fMRI scans were collected using a 3T MRI scanner (Tim Trio, Siemens Medical, Erlangen, Germany) equipped with a 12channel head coil. Whole-brain $\mathrm{FMRI}$ data were acquired using a spin echo, T2*-weighted BOLD pulse sequence (TR $=2000 \mathrm{~ms}$, $\mathrm{TE}=40 \mathrm{~ms}, \mathrm{FOV}=200 \times 200 \mathrm{~mm}$, matrix $=64 \times 64$, in-plane resolution $3.125 \mathrm{~mm}, 23$ axial slices, slice thickness $=5 \mathrm{~mm}, 180$ volumes). The fMRI task consisted of presentation of blocks of images adapted from the International Affective Picture System (IAPS [59]), comprising a mild visual stress challenge. The task had an approximate duration of 18 min (3 runs, 6 min each). Each run contained non-randomized blocks of negative (high arousal/ negative valence), neutral (low arousal/neutral valence) and fixation images (Fourier transform of neutral images). Each run consisted of 12 blocks, four each of negative, neutral, and fixation trials. Each block consisted of six images, each presented for $5 \mathrm{~s}$. After the fMRI session, participants were shown two additional blocks of stimuli and reported their subjective evaluation of image valence and arousal using the Self-assessment Manikin (SAM [59]). This task has been previously used by our group in multiple studies with demonstrated reliability and validity to invoke HPA-related hormonal, autonomic, neural responses, and behavior $[5,9,12,48]$.

\section{Analysis of $\mathrm{fMRI}$ data}

As reported in [46, 47], fMRI data were preprocessed and analyzed using Statistical Parametric Mapping (SPM8) [60] and included non-linear volume-based spatial normalization (MNI152 brain template), spatial smoothing with a Gaussian filter $(6 \mathrm{~mm}$ at FWHM, which was then re-sampled to $3 \mathrm{~mm}$ isotropic), high pass filtering (180 s) to control for low frequency scanner drift and artifact detection using an artifact detection toolbox (ART) to identify outliers in the global mean image time series (threshold: 3.5 standard deviations from the mean) and movement (threshold: $0.7 \mathrm{~mm}$, measured as scan-to-scan movement, separately for translation and rotation). Outliers were entered as nuisance regressors in the first-level, single-subject GLM analysis. Each epoch of trials was included in a general linear model (GLM) as a separate boxcar function convolved with a canonical hemodynamic response basis function. Specific comparisons of interest (negative > neutral BOLD signal changes) from the first-level, were then tested using linear contrasts and SPM t-maps were created. Contrast images from the first-level analyses were submitted to a second-level random effects analysis.

BOLD response analysis

Statistical maps comparing negative $>$ neutral stimuli across the full sample were generated at voxel-wise family-wise (FWE)- corrected $p<0.05[46,47]$. Next, an intersection analysis was performed using the MarsBaR toolbox to identify clusters that were conjointly located within anatomic boundaries of stress response circuitry ROIs (PAG, HYPO, AMYG, HIPP, OFC, ACC, and $\mathrm{mPFC}$ ). Average parameter estimates (percent signal change values) within each ROI were extracted for each participant using REX [61] and were included in a GLM stratified by sex to assess the modulatory role of depressed mood on the relationship between BOLD response to negative stimuli in the stress response circuitry and cardiovagal activity (HFn). For the evaluation of interactions with depressed mood, POMS depression/dejection subscale scores were included in the model and regression coefficients were calculated for subjects with high (mean +1 SD) and low (mean $-1 \mathrm{SD}$ ) levels of depressive symptoms. Statistical analyses were performed using STATA (StataCorp, College Station TX). An additional post hoc whole-brain analysis was performed to evaluate mood- and sex-dependent interactions on the relationship between BOLD responses and cardiovagal response to negative stimuli in areas outside our stress response circuitry ROls (see supplementary material).

Assessment of functional connectivity

A task-related connectivity using generalized psychophysiological interaction (gPPI; [62]) was conducted. HYPO and AMYG were used as seeds, given their well-known importance in cardiac autonomic function regulation. The extracted fMRI time series from these seed ROls (defined as: clusters identified from the intersection analyses in MarsBaR described above) were added to two additional regressors (interaction of the seed fMRI time series with the regressors for negative and neutral content) to individual subject-level GLMs. Connectivity was determined at the singlesubject level by estimating the difference between the interaction of the seed time series with the regressor for negative compared with the neutral stimuli. Results of the first-level analysis were then entered into second-level random effects analysis to evaluate group-level differences in connectivity during exposure to negative versus neutral content.

Given hypotheses about specific brain regions in the stress response circuitry, the small volume correction (SVC) approach in SPM8 was used, limiting voxel-wise analyses to voxels within a priori ROls. Target ROIs (PAG, HYPO, AMYG, HIPP, OFC, ACC, mPFC) were defined as described above for the BOLD response analysis and implemented as overlays on the SPM8 canonical brain using the Wake Forest University Pick Atlas ROI toolbox for SPM [63]. Average connectivity values (beta weights of PPI regressors) in significant target clusters were extracted using REX [61]. We then used a GLM analyses stratified by sex to assess the modulatory role of depressed mood on the association between cardiovagal activity and HYPO and AMYG connectivity with other regions from the stress response circuitry in a similar manner as described above for BOLD response.

\section{RESULTS}

Demographic and clinical characteristics

Fifty subjects (age: $45.5 \pm 5.0$ years old; 28 females, 22 males) were included in the study. Twenty were diagnosed with recurrent major depressive disorder in remission (MDD; 10 females, 10 males), 14 subjects with psychoses (PSY; 9 females, 5 males), and 16 were healthy controls (CTRL; 9 females, 7 males). Demographics and clinical characteristics of the sample are in Table 1. There were no significant differences between men and women in age, ethnicity, handedness, BMI, parental SES, educational level, the rate of DSM diagnoses or the proportion taking psychotropic medications. A significant difference was found in the duration of illness and the WAIS vocabulary scores, being significantly greater in men compared with women (Table 1). Men also presented significantly higher scores for the POMS subscales tension/anxiety, 
Table 1. Demographics and clinical characteristics of the sample.

\begin{tabular}{|c|c|c|c|}
\hline & Females $(n=28)$ & Males $(n=22)$ & $p$ value \\
\hline Ethnicity (\%): Caucasian ( $n$ ) & $92.9 \%(26 / 28)$ & $95.4 \%(21 / 22)$ & n.s. \\
\hline $\begin{array}{l}\text { Handedness (\%): right-handed } \\
(n)\end{array}$ & $92.9 \%(26 / 28)$ & $86.3 \%(19 / 22)$ & n.s. \\
\hline Age in years: $M(S D)$ & $45.1(5.5)$ & $46.1(4.3)$ & n.s. \\
\hline BMI: M (SD) & $29.9(7.1)$ & $28.4(4.5)$ & n.s. \\
\hline \multicolumn{4}{|l|}{ Education level } \\
\hline $\begin{array}{l}\text { \% Without completed high } \\
\text { school }(n)\end{array}$ & $3.7 \%(1 / 28)$ & $9.5 \%(2 / 22)$ & n.s. \\
\hline$\%$ Completed high school $(n)$ & $22.2 \%(6 / 28)$ & $23.81 \%(5 / 22)$ & \\
\hline$\%$ More than high school $(n)$ & $71.4 \%(20 / 28)$ & $63.6 \%(14 / 22)$ & \\
\hline$\%$ Missing $(n)$ & $3.6 \%(1 / 28)$ & $4.5 \%(1 / 22)$ & \\
\hline $\begin{array}{l}\text { WAIS vocabulary, age-scaled: M } \\
\text { (SD) }\end{array}$ & $10.0(3.47)$ & $12.1(3.36)$ & 0.03 \\
\hline $\begin{array}{l}\text { WAIS block design, age- } \\
\text { scaled: } M \text { (SD) }\end{array}$ & 9.7 (3.08) & $10.7(2.34)$ & n.s. \\
\hline \multicolumn{4}{|l|}{ DSM-based diagnosis } \\
\hline$\%$ MDD in remission $(n)$ & $35.7 \%(10 / 28)$ & $45.4 \%(10 / 22)$ & n.s. \\
\hline \% Psychosis $(n)$ & $32.1 \%(9 / 28)$ & $22.7 \%(5 / 22)$ & \\
\hline$\%$ Healthy controls $(n)$ & $32.1 \%(9 / 28)$ & $31.8 \%(7 / 22)$ & \\
\hline $\begin{array}{l}\text { Age at symptom onset in } \\
\text { years: } M \text { (SD) }\end{array}$ & $26.0(8.86)$ & $20.7(7.83)$ & n.s. \\
\hline $\begin{array}{l}\text { Duration of illness in years: } \\
M(S D)\end{array}$ & $17.9(8.86)$ & $24.9(7.66)$ & 0.02 \\
\hline $\begin{array}{l}\text { Psychotropic medication: } \\
\% \text { on psychotropic medication } \\
(n)\end{array}$ & $35.7 \%(10 / 28)$ & $31.8 \%(7 / 22)$ & n.s. \\
\hline
\end{tabular}

\begin{tabular}{|c|c|c|c|c|c|}
\hline \multirow[t]{2}{*}{ Measured variable } & \multicolumn{2}{|c|}{$\begin{array}{l}\text { Females } \\
(n=28)\end{array}$} & \multicolumn{2}{|c|}{$\begin{array}{l}\text { Males } \\
(n=22)\end{array}$} & \multirow[t]{2}{*}{$p$ value } \\
\hline & M & SD & M & SD & \\
\hline \multicolumn{6}{|l|}{ Self-assessment manikin (SAM) } \\
\hline Arousal during neutral stimuli & 8.33 & 1.46 & 8.27 & 0.92 & n.s. \\
\hline Arousal during negative stimuli & 4.57 & 2.28 & 4.95 & 2.40 & n.s. \\
\hline Valence of negative stimuli & 7.62 & 1.50 & 7.75 & 1.20 & n.s. \\
\hline Valence of neutral stimuli & 4.16 & 1.13 & 4.52 & 0.94 & n.s. \\
\hline \multicolumn{6}{|l|}{ State-Trait Anxiety Inventory (STAI) } \\
\hline State Anxiety & 32.1 & 8.67 & 32.2 & 8.03 & n.s. \\
\hline Trait Anxiety & 37.0 & 9.81 & 40.7 & 11.5 & n.s. \\
\hline \multicolumn{6}{|l|}{ POMS } \\
\hline Tension/anxiety & 30.6 & 1.70 & 33.0 & 4.16 & 0.01 \\
\hline Depression/dejection & 33.7 & 2.39 & 37.2 & 4.10 & $<0.01$ \\
\hline Anger/hostility & 37.7 & 1.23 & 39.2 & 2.83 & 0.01 \\
\hline Vigor/activity & 59.8 & 10.2 & 55.4 & 11.1 & n.s. \\
\hline Fatigue/inertia & 37.4 & 4.82 & 42.3 & 5.75 & $<0.01$ \\
\hline Confusion/bewilderment & 31.0 & 2.09 & 33.6 & 2.47 & $<0.01$ \\
\hline
\end{tabular}

depression/dejection, anger/hostility, fatigue/inertia, and confusion/bewilderment (Table 2).

No significant differences were found between men and women for the percent change in the HFn index $(3.83 \pm 20.9$ vs $0.19 \pm 18.6 \%, p=0.5)$. An analysis stratified by sex revealed women with higher depressed mood scores presented a significant reduction in cardiovagal activity during negative affective stimuli compared with those with low levels of depressed
$\operatorname{mood}(\mathrm{HFn}=-7.36 \pm 20.3$ vs $8.44 \pm 13.1 \%, p=0.03$ ), whereas no significant differences in HFn were found between men with high and low levels of depressed mood.

Effects of depressed mood and sex on the relationship between stress response circuitry activation and cardiovagal activity A multivariate general linear model stratified by sex revealed that depressed mood significantly modulated the relationship between R AMYG activity and cardiovagal response to negative affective stimuli in women. Results showed that women presented a significant interaction between depressed mood and the association of HFn changes and BOLD response in R AMYG ( $F$ $(3,22)=3.13, p=0.04)$. Further analyses revealed that women with high depressed mood scores exhibited an association between reduction of cardiovagal activity and increased R AMYG activation $\left(\beta=-0.027, t(22)=-2.75, p=0.013\right.$, Adj $\left.R^{2}=0.22\right)$, whereas no significant associations were found in either women with low levels of depressed mood or men with either high or low levels of depressed mood (Fig. 1). The analyses also revealed a significant interaction between depressed mood and the association of cardiovagal modulation and HYPO activity during exposure to negative images in women $(F(3,22)=5.40, p<0.01)$. Similar to findings for R AMYG, women with high levels of depressed mood exhibited an inverse relationship between cardiovagal activity (HFn difference) and HYPO activation $(\beta=-0.025, t(22)=-3.76$, $p=0.001$, $\operatorname{Adj} R^{2}=0.37$ ), whereas no significant effects were observed in those with low levels of depressed mood or men with high or low depressed mood scores (Fig. 2).

To explore additional regions outside of the hypothesized stress circuitry, a post hoc whole-brain analysis revealed that women with higher depressed mood scores had a significant association between low cardiovagal response and increased activation of posterior cingulate cortex (PCC) $(\beta=-0.012, t(22)=-2.41, p=$ 0.026 , $\left.\operatorname{Adj} R^{2}=0.24\right)$ (Fig. S2) and left insula (INS) $(\beta=-0.021$, $t(22)=-3.17, p=0.005$, Adj $\left.R^{2}=0.28\right)$ (Fig. S3). This analysis did not reveal significant associations in women with low levels of depressed mood or men with high or low depressed mood scores, although we did not have the statistical power in this post hoc analysis to test for multiple interaction effects with sex.

Higher cortisol levels after the stress task challenge (min 90) were associated significantly with lower cardiovagal activity $(r=$ $-0.34, p=0.01$ ) (Fig. 3). There were no interactions with depressed mood or sex for this association.

Effects of depressed mood and sex on the relationship between stress response circuitry functional connectivity and cardiovagal modulation

To further extend our findings, we used AMYG and HYPO as seeds and tested their connectivity with the other regions of interest in the stress response circuitry. While there was no main effect of HYPO or AMYG connectivity on cardiovagal variability, we found that women exhibited significant interactions of depressed mood with the association of cardiovagal activity and connectivity between HYPO and right OFC $(F(3,22)=3.95, p=0.03)$ and AMYGright HIPP $(F(3,22)=3.33, p=0.04)$. Post hoc analyses revealed that in women with high levels of depressed mood, lower connectivity between HYPO and right OFC $(\beta=0.028, t(22)=2.52$, $p=0.02, \operatorname{Adj} R^{2}=0.17$ ) (Fig. 4) and right AMYG-right HIPP $(\beta=$ $\left.0.013, t(22)=3.07, \quad p=0.01, \quad \operatorname{Adj} R^{2}=0.23\right)$ were significantly associated with lower cardiovagal response to negative affective stimuli. No significant relationship was identified in women with low levels of depressed mood or men with high or low depressed mood scores.

\section{DISCUSSION}

This study investigated the impact of depressed mood and sex on the coupling between neural and cardiac autonomic responses to 


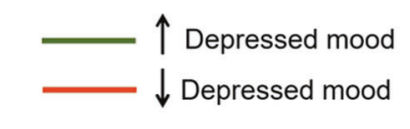

Females

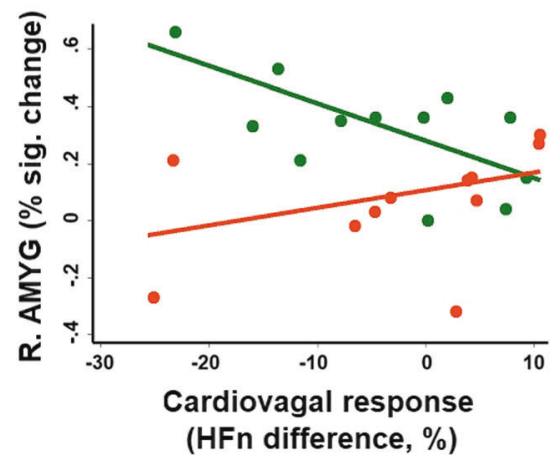

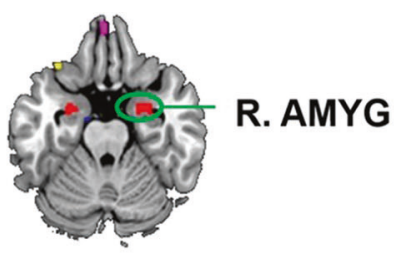

Males

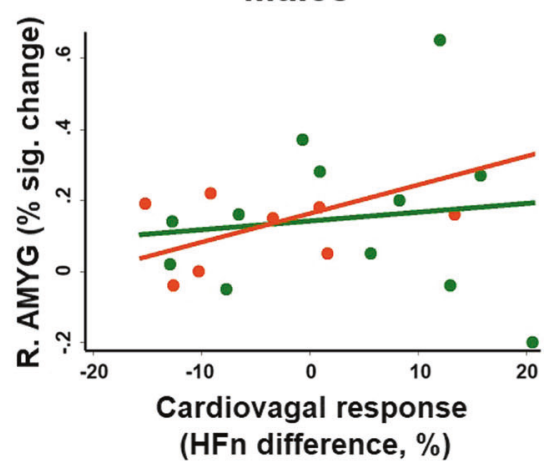

Fig. 1 Impact of depressed mood and sex on the relationship between right AMYG activity and cardiovagal regulation in response to negative affective stimuli (HFn difference with neutral images). Women with high depressed mood scores presented a significant negative relationship between cardiovagal response and R AMYG activity $\left(\beta=-0.027, t(22)=-2.75, p=0.013\right.$, Adj $\left.R^{2}=0.22\right)$. No significant association was found between cardiovagal response and right AMYG Activity in men with high depressed mood scores $(\beta=0.0008, t(19)=0.14, p=0.88$, $\left.\operatorname{Adj} R^{2}=0.14\right)$.

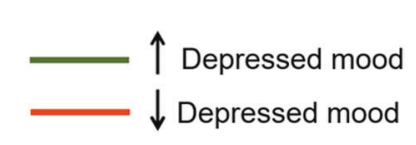

Females

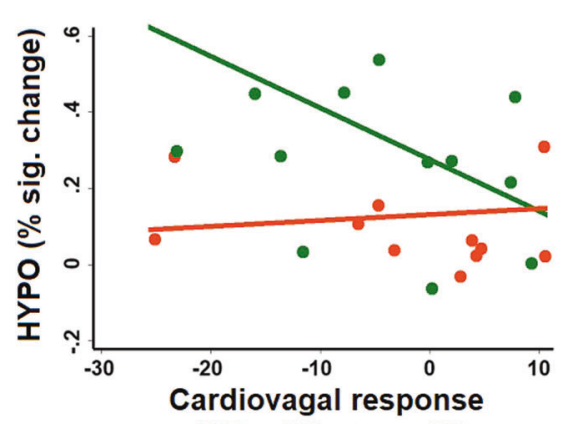

(HFn difference, \%)

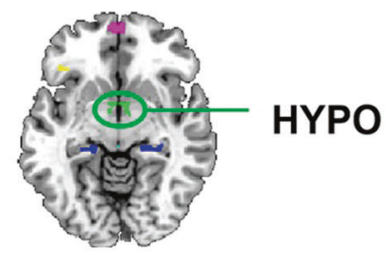

Males

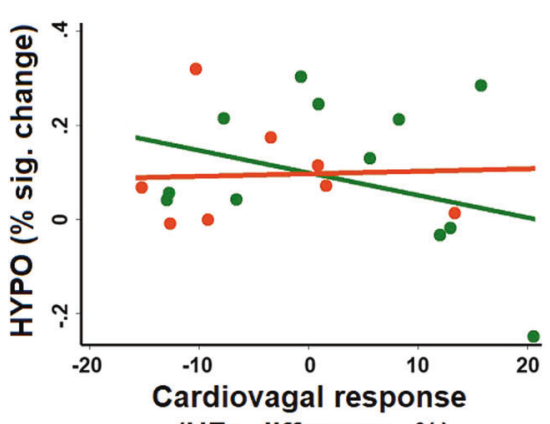

(HFn difference, \%)

Fig. 2 Impact of depressed mood and sex on the relationship between HYPO activity and cardiovagal regulation in response to negative affective stimuli (HFn difference with neutral images). Women with high depressed mood scores presented an inverse relationship between cardiovagal response (HFn) and HYPO activation $\left(\beta=-0.025, t(22)=-3.76, p=0.001\right.$, Adj $\left.R^{2}=0.37\right)$. No significant association was found between cardiovagal response and HYPO activity in men with high depressed mood scores $\left(\beta=-0.003, t(19)=-0.93, p=0.36, \mathrm{Adj} R^{2}=0.19\right)$.

negative affective stimuli across a sample of individuals with major psychiatric disorders and healthy controls. Our results revealed that in women with high levels of depressed mood, greater activation of R AMYG and HYPO and lower connectivity of HYPO-OFC and R AMYG-HIPP were associated with reduction of cardiac parasympathetic activity (lower HFn) in response to negative affective stimuli, regardless of diagnostic status. Findings in this study extend our previous results from this population $[46,47]$ demonstrating significant associations between lower HYPO-HIPP connectivity and increased cortisol response in women with high levels of depressed mood. Not surprising, cortisol response was significantly associated with cardiovagal response, such that high $\mathrm{HF}$, suggesting higher parasympathetic cardiac tone, was associated with lower cortisol response to negative stimuli. Taken together, our results suggest that depressed mood, particularly among women, is significantly associated with maladaptive brain activity responses to negative stressful stimuli and physiological alterations characterized by adrenal and autonomic dysregulation. Dysregulation of autonomic nervous system function has been associated with higher risk for CVD [3]. Thus, findings in the study reported here suggest neural and physiologic substrates that may be associated with the 
greater prevalence of comorbid mood disorders and CVD in women than men $[3,7]$.

Our results demonstrating an association between higher amygdala activation and parasympathetic cardiovagal activity reduction in response to negative affective stimuli are consistent with previous research $[64,65]$. The amygdala is involved in the regulation of physiological responses during emotional arousal via its direct connections with the HPA axis [66]. Furthermore, the amygdala is involved with regulation of both parasympathetic and sympathetic cardiac control [64, 65]. Neurophysiological studies have consistently demonstrated that amygdala activity is correlated with cardiac autonomic response during stressful tasks involving cognitive or affective stimuli [64, 67], and human and animal studies have revealed that subjects with amygdalar lesions present dampened cardiac autonomic responses to emotional stress [68].

In addition, our findings of a significant relationship between hypothalamic activation and reduction of parasympathetic cardiovagal modulation in response to negative affective stimuli are also consistent with previous research [64]. The hypothalamus,

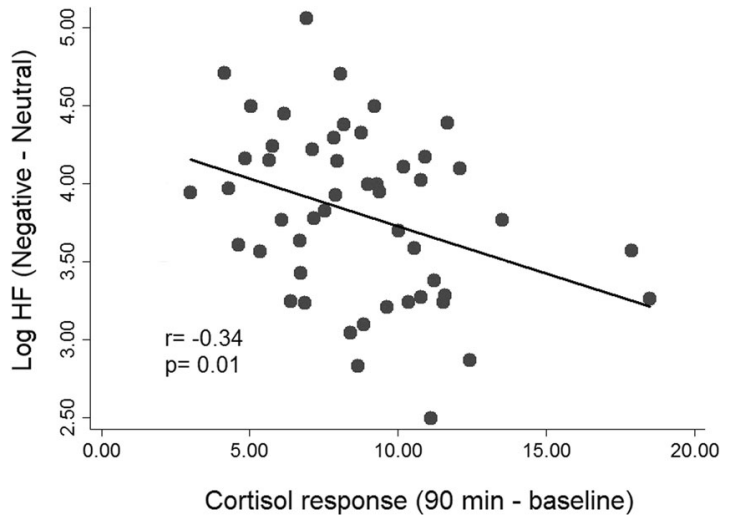

Fig. 3 Correlation between cortisol response and cardiovagal response (Log HF power) to negative affective stimuli. Higher cortisol levels after the stress task challenge were significantly associated with lower cardiovagal activity $(r=-0.34, p=0.01)$ in the subjects included in the study. and in particular the paraventricular nucleus (PVN), is a key relay station for the integration of neuroendocrine, autonomic, vascular, and inflammatory responses to stress [7]. Within its anatomic boundaries the PVN contains neurons that project to autonomic centers in the brainstem, including NTS and medullary efferent (premotor) parasympathetic nuclei, such as the nucleus ambiguus, regulating cardiac pacemaker activity via myelinated vagal pathways $[28,31]$.

Our findings implicating limbic system regions, such as amygdala and hypothalamus, in ANS modulation, are not surprising, given that they are also critical in mood regulation. In fact, in a previous report with a larger sample of this study population, our group identified a positive association between dysphoric mood and BOLD response in the hypothalamus and right amygdala, with sexdependent analyses showing a significant effect in women compared with men [46]. Our current study expands these results by revealing that the hypothalamic and amygdala activation in women with depressed symptomatology is significantly associated with reduction of parasympathetic cardiac modulation and higher cortisol levels in response to negative affective stimuli.

An additional post hoc whole-brain analysis identified some regions (PCC, INS) outside our stress response circuitry regions of interest that were significantly associated with cardiovagal activity in women with higher depressed mood scores and not men. The posterior cingulate and insula have been previously identified as key areas of the central autonomic network responsible for the regulation of parasympathetic and sympathetic activity [64]. Studies have shown structural and functional abnormalities of these areas in depressed subjects [69-72], which have been associated with increased rumination and altered interoceptive and visceroceptive processing in $\operatorname{MDD}[70,72,73]$. Given that these results were revealed in a post hoc analysis and we did not have the statistical power to test for multiple interaction effects, further studies are needed to confirm their sex-dependent role in the dysregulation of cardiovagal response associated with MDD. However, the fact that the sex-dependent effects were consistent with our findings on the hypothesized stress circuitry regions of interest underscores their validity.

Overall, our results support a significant role of hyperactivity of subcortical arousal regions as a driver of greater vagal activity
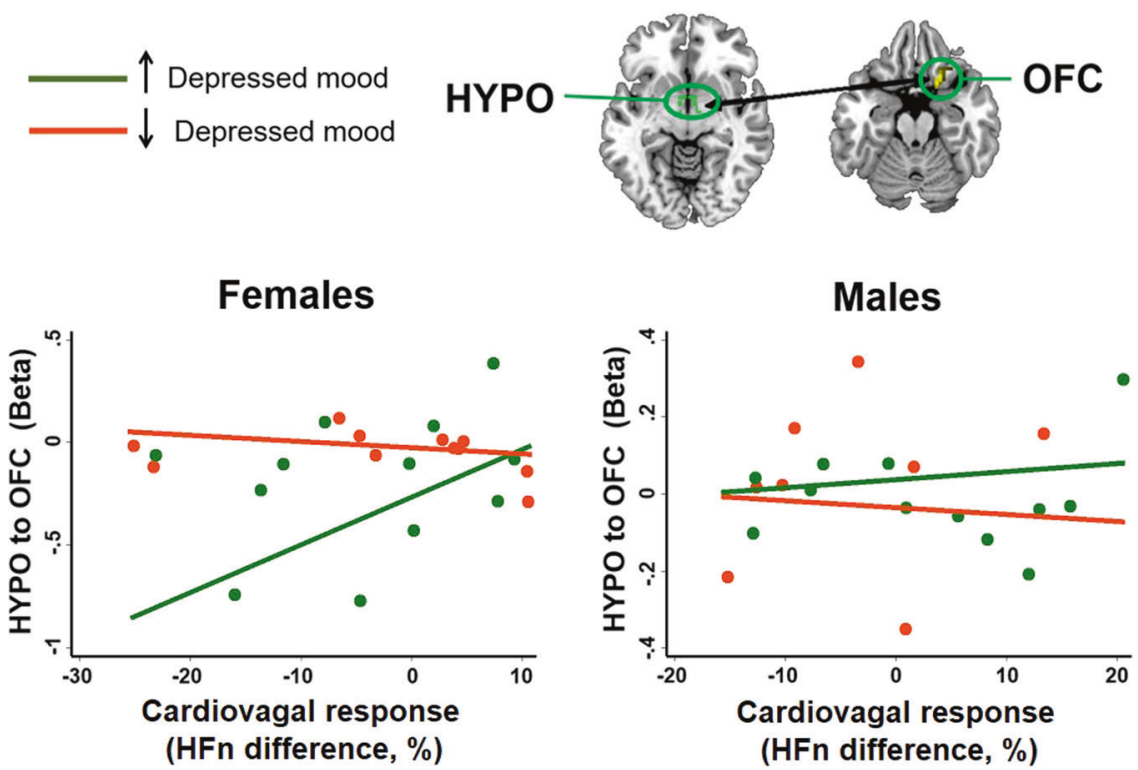

Fig. 4 Impact of depressed mood and sex on the relationship between HYPO to OFC connectivity and cardiovagal regulation in response to negative affective stimuli (HFn difference with neutral images). Women with high depressed mood scores presented a direct relationship between cardiovagal response and HYPO connectivity to right OFC $\left(\beta=0.028, t(22)=2.52, p=0.02\right.$, Adj $\left.R^{2}=0.17\right)$. No significant association was found between cardiovagal response and HYPO connectivity to right OFC in men with high depressed mood scores $(\beta=0.0015, t(19)=$ $\left.0.29, p=0.77, \operatorname{Adj} R^{2}=0.01\right)$. 
reduction during negative emotional arousal associated with depressive symptomatology. Our findings also showed a reduced OFC-HYPO and R AMYG-HIPP connectivity correlated with low HFn power in these subjects, i.e., low parasympathetic activity, suggesting lack of cortical inhibitory control as one of the mechanisms responsible for increased subcortical excitability and dysregulation of vagal response to negative emotional stimuli in this population. The orbitofrontal cortex has been generally associated with the regulation of autonomic and visceral aspects of emotional responses, as well as an integrative function between these states and goal directed behavior $[74,75]$. It has a high density of mineralocorticoid and glucocorticoid receptors and is involved in the inhibitory regulation of stress-mediated HPA axis activity and modulation of cardiovagal activity [76].

There is a large body of evidence demonstrating orbitofrontal cortex alterations in patients with affective disorders and schizophrenia [77], and previously our group reported the presence of reduced OFC-HYPO connectivity in female subjects with dysphoric mood [46]. Thus, it is not surprising that we found a relationship between reduction of OFC-HYPO connectivity and lower cardiovagal response in women with depressed symptoms included in our study. In addition, we observed an association between lower HIPP-R AMYG connectivity and lower parasympathetic activity in women with high levels of depressive mood. The hippocampus has a critical role in the negative feedback to the PVN in the regulation of HPA-axis and central regulation of ANS activity [78]. In a similar manner to the orbitofrontal cortex, hippocampal alterations have been involved in the pathogenesis of mood and stress-related disorders $[4,77,79]$. In patients with these conditions, threat perception is associated with dysregulation of hippocampal circuits leading to maladaptive endocrine and autonomic responses [80]. The HIPP-AMYG neural connection is particularly relevant to the integration of neurovisceral information and top-down regulation of cardiorespiratory and autonomic control during emotional responses [81, 82]. A reduced connectivity between these two regions may lead to impaired cardiovagal modulation in response to negative affective stimuli, as exhibited by women with depressed mood in our study.

Importantly, the associations reported here between the stress response circuitry and parasympathetic dysregulation in response to negative affective stimuli was only significant in women in our sample. These findings are consistent with previous studies documenting functional and anatomical basis for sex differences in the central mechanisms for autonomic regulation [7, 83]. For example, in a study using PET and HRV measurements in healthy controls, Nuget et al. [83] identified a significant positive correlation between regional cerebral blood flow ( $\mathrm{rCBF}$ ) in the amygdala and parasympathetic indices in women, while men exhibited negative correlations. Our results extend these findings by demonstrating a significant interaction of depressive mood with sex in the neural mechanisms controlling parasympathetic response to negative stress. Differential associations of the stress response circuitry and cardiovagal indices between men and women may reflect known sexual dimorphisms in the hypothalamus and amygdala, as well as hippocampus and orbitofrontal cortex [15]. Further, our group has previously shown that developmental alterations of normal sexual dimorphisms of this circuitry may be associated with sex differences in abnormal neuroendocrine and autonomic responses to negative stress in adulthood that may predispose for sex-dependent risk to develop mood and psychotic disorders [84] and comorbidity with cardiovascular disease $[2,3,7]$.

There are a few potential limitations to this study. First, the methodology used in the study does not allow us to disentangle afferent from efferent contributions to the associations between brain and cardiovagal responses. Future studies should address whether the observed associations between stress response circuitry activity and autonomic indices reflect a modulatory effect from brain to heart or vice versa. Another limitation of the study is related with the inclusion of a mixed sample of participants with a limited severity of active depressive symptomatology, given that the evaluated subjects had recurrent psychiatric illness in remission. Thus, our findings may reflect the effects of long-standing mood dysregulation rather than current clinical state. Even so, the fact that a significant relationship between depressive symptoms and cardiac and brain measures was found only in the women could be interpreted as underscoring support of our hypothesis of sex-dependent interactions. Further, we would expect that the observed relationships may be even more pronounced in subjects in an active depressive episode. Finally, our findings suggest that we found greater severity of mood symptomatology in men compared with women in our sample. Although unexpected, the fact that we found significant associations between our variables of interest among the women, despite higher depressed mood scores in the men, provides additional support for the validity of our findings in women and underscores the sex differences reported.

In summary, our findings revealed mood and sex-dependent interactions in the association between stress response circuitry activity and parasympathetic cardiovagal modulation in response to negative affective stimuli. Women with higher depressive mood showed maladaptive brain responses associated with reduced cardiovagal responses during negative emotional arousal. Although epidemiological studies have revealed substantial sex differences in the incidence of comorbidity between mental disorders and cardiovascular disease with major public health implications worldwide, the pathophysiology underlying these associations still remain unclear. We propose here that the evaluation of the mechanisms linking these conditions should be informed by brain and physiology associations and the inclusion of a sex-dependent lens that may be shared across psychiatric disorders in order to develop more precise and efficacious therapeutics targeted to comorbidities. For example, we are currently developing a novel transcutaneous vagal neurostimulation device [85] targeted to mood and cardiovagal activity in people with major depression and cardiometabolic disorders informed using a sex differences lens, by translating findings, such as those presented here, to select sex-dependent target engagement in the brain and associated physiology for optimization of the technology.

\section{FUNDING AND DISCLOSURE}

This work was supported by NIMH R03 MH105585 (with original data collected in ORWH-NIMH P50 MH082679 and NIMH R01 MH56956 Phase III; JMG PI) and a philanthropic award to JMG from Ms. Gwill York, and NIMH R21 MH103468 (JMG \& VN, multi-PIs). The research was also conducted with support from Harvard Catalyst and the Harvard Clinical and Translational Science Center (NIH \#UL1 RR025758). RG was supported by a NARSAD Young investigator Grant from the Brain \& Behavior Research Foundation (Grant no. 26236). For KM's time, we would like to thank the European Union who financed the Marie Curie Intra-European Fellowship for Career Development and The Ministry of Education, Youth and Sports of the Czech Republic/MEYS (CEITEC 2020; LQ1601). VN's time was also supported by $\mathrm{NIH}$, Office Of The Director (OT2-OD023867). Currently, Drs. Goldstein and Napadow are on the scientific advisory board of Cala Health and Dr. Goldstein has equity in Cala Health. The study presented here was conducted prior to that relationship. The authors declare no competing interests.

\section{ACKNOWLEDGEMENTS}

We would like to thank Harlyn Aizley, Ed.M., Anne Remington, M.A., Jennifer Walch, M.Ed., Sara Cherkerzian, Sc.D., and Brandon Fluegel for their substantial contributions 
to the collection and management of data from the original cohort studies associated with the sample.

\section{ADDITIONAL INFORMATION}

Supplementary Information accompanies this paper at (https://doi.org/10.1038/ s41386-020-0651-x)

Publisher's note Springer Nature remains neutral with regard to jurisdictional claims in published maps and institutional affiliations.

\section{REFERENCES}

1. García-Gómez RG, López-Jaramillo P, Tomaz C. The role played by the autonomic nervous system in the relation between depression and cardiovascular disease. Rev de neurologia. 2007:44:225-33.

2. Goldstein JM, Holsen L, Handa R, Tobet S. Fetal hormonal programming of sex differences in depression: linking women's mental health with sex differences in the brain across the lifespan. Front Neurosci. 2014;8:247.

3. Goldstein JM, Handa RJ, Tobet SA. Disruption of fetal hormonal programming (prenatal stress) implicates shared risk for sex differences in depression and cardiovascular disease. Front Neuroendocrinol. 2014;35:140-58.

4. Goldstein JM, Lancaster K, Longenecker JM, Abbs B, Holsen LM, Cherkerzian S, et al. Sex differences, hormones, and fMRI stress response circuitry deficits in psychoses. Psychiatry Res. 2015;232:226-36.

5. Goldstein JM, Jerram M, Abbs B, Whitfield-Gabrieli S, Makris N. Sex differences in stress response circuitry activation dependent on female hormonal cycle. J Neurosci. 2010;30:431-38.

6. Holsen LM, Spaeth SB, Lee J-H, Ogden LA, Klibanski A, Whitfield-Gabrieli S, et al. Stress response circuitry hypoactivation related to hormonal dysfunction in women with major depression. J Affect Disord. 2011;131:379-87.

7. Goldstein JM, Hale T, Foster SL, Tobet SA, Handa RJ. Sex differences in major depression and comorbidity of cardiometabolic disorders: impact of prenatal stress and immune exposures. Neuropsychopharmacology. 2019;44:59-70.

8. Tobet SA, Handa RJ, Goldstein JM. Sex-dependent pathophysiology as predictors of comorbidity of major depressive disorder and cardiovascular disease. Pflug Arch: Eur J Physiol. 2013;465:585-94.

9. Holsen LM, Lancaster K, Klibanski A, Whitfield-Gabrieli S, Cherkerzian S, Buka S, et al. HPA-axis hormone modulation of stress response circuitry activity in women with remitted major depression. Neuroscience. 2013;250:733-42.

10. Mayberg HS. Limbic-cortical dysregulation: a proposed model of depression. J neuropsychiatry Clin Neurosci. 1997;9:471-81.

11. Dougherty D, Rauch SL. Neuroimaging and neurobiological models of depression. Harv Rev Psychiatry. 1997;5:138-59.

12. Goldstein JM, Jerram M, Poldrack R, Ahern T, Kennedy DN, Seidman LJ, et al. Hormonal cycle modulates arousal circuitry in women using functional magnetic resonance imaging. J Neurosci. 2005;25:9309-16.

13. Tobet SA, Hanna IK. Ontogeny of sex differences in the mammalian hypothalamus and preoptic area. Cell Mol Neurobiol. 1997;17:565-601.

14. Tobet S, Knoll JG, Hartshorn C, Aurand E, Stratton M, Kumar P, et al. Brain sex differences and hormone influences: a moving experience? J Neuroendocrinol. 2009;21:387-92.

15. Goldstein JM, Seidman LJ, Horton NJ, Makris N, Kennedy DN, Caviness VS, et al Normal sexual dimorphism of the adult human brain assessed by in vivo magnetic resonance imaging. Cereb Cortex. 2001;11:490-97.

16. McEwen BS. Gonadal steroid influences on brain development and sexual differentiation. Int Rev Physiol. 1983;27:99-145.

17. Domes G, Schulze L, Bottger M, Grossmann A, Hauenstein K, Wirtz PH, et al. The neural correlates of sex differences in emotional reactivity and emotion regulation. Hum Brain Mapp. 2010;31:758-69.

18. Whittle S, Yucel M, Yap MB, Allen NB. Sex differences in the neural correlates of emotion: evidence from neuroimaging. Biol Psychol. 2011;87:319-33.

19. Goldstein JM, Cherkerzian S, Buka SL, Fitzmaurice G, Hornig M, Gillman M, et al. Sex-specific impact of maternal-fetal risk factors on depression and cardiovascular risk 40 years later. J Dev Orig Health Dis. 2011;2:353-64.

20. Gilman SE, Cherkerzian S, Buka SL, Hahn J, Hornig M, Goldstein JM. Prenatal immune programming of the sex-dependent risk for major depression. Transl Psychiatry. 2016;6:e822.

21. Barefoot JC, Helms MJ, Mark DB, Blumenthal JA, Califf RM, Haney TL, et al. Depression and long-term mortality risk in patients with coronary artery disease. Am J Cardiol. 1996;78:613-7.

22. Kawachi I, Sparrow D, Vokonas PS, Weiss ST. Symptoms of anxiety and risk of coronary heart disease. The Normative Aging Study. Circulation 1994;90:2225-9.
23. Everson SA, Kaplan GA, Goldberg DE, Salonen R, Salonen JT. Hopelessness and 4year progression of carotid atherosclerosis; the Kuopio Ischemic Heart Disease Risk Factor Study. Arteriosclerosis, Thrombosis, Vasc Biol. 1997;17:1490-95.

24. Glassman AH, Shapiro PA. Depression and the course of coronary artery disease. Am J Psychiatry. 1998;155:4-11.

25. Jones DJ, Bromberger JT, Sutton-Tyrrell K, Matthews KA. Lifetime history of depression and carotid atherosclerosis in middle-aged women. Arch Gen Psychiatry. 2003;60:153-60.

26. Scherrer JF, Xian H, Bucholz KK, Eisen SA, Lyons MJ, Goldberg J, et al. A twin study of depression symptoms, hypertension, and heart disease in middle-aged men. Psychosom Med. 2003;65:548-57.

27. Krishnan KR, Doraiswamy PM, Clary CM. Clinical and treatment response characteristics of late-life depression associated with vascular disease: a pooled analysis of two multicenter trials with sertraline. Prog Neuro-Psychopharmacol Biol Psychiatry. 2001;25:347-61.

28. Ferguson AV, Latchford KJ, Samson WK. The paraventricular nucleus of the hypothalamus-a potential target for integrative treatment of autonomic dysfunction. Expert Opin Ther Targets. 2008;12:717-27.

29. Jankord R, Herman JP. Limbic regulation of hypothalamo-pituitary-adrenocortical function during acute and chronic stress. Ann N. Y. Acad Sci. 2008;1148:64-73.

30. Tsigos C, Chrousos GP. Hypothalamic-pituitary-adrenal axis, neuroendocrine factors and stress. J Psychosom Res. 2002;53:865-71.

31. Ciriello J, McMurray JC, Babic T, de Oliveira CVR. Collateral axonal projections from hypothalamic hypocretin neurons to cardiovascular sites in nucleus ambiguus and nucleus tractus solitarius. Brain Res. 2003;991:133-41.

32. Chitravanshi VC, Kawabe K, Sapru HN. GABA and glycine receptors in the nucleus ambiguus mediate tachycardia elicited by chemical stimulation of the hypothalamic arcuate nucleus. Am J Physiol Heart Circ Physiol. 2015;309:H174-84.

33. Nemeroff CB, Widerlöv E, Bissette G, Walléus H, Karlsson I, Eklund K, et al. Elevated concentrations of CSF corticotropin-releasing factor-like immunoreactivity in depressed patients. Science 1984;226:1342-44.

34. Arborelius L, Owens MJ, Plotsky PM, Nemeroff CB. The role of corticotropinreleasing factor in depression and anxiety disorders. J Endocrinol. 1999;160:1-12.

35. Evans DL, Nemeroff CB. The clinical use of the dexamethasone suppression test in DSM-III affective disorders: correlation with the severe depressive subtypes of melancholia and psychosis. J Psychiatr Res. 1987;21:185-94.

36. Oquendo MA, Echavarria G, Galfalvy HC, Grunebaum MF, Burke A, Barrera A, et al. Lower cortisol levels in depressed patients with comorbid post-traumatic stress disorder. Neuropsychopharmacology. 2003;28:591-98.

37. Barden N. Implication of the hypothalamic-pituitary-adrenal axis in the physiopathology of depression. J Psychiatry Neurosci. 2004;29:185-93.

38. Goldstein JM, Holsen L, Cherkerzian S, Misra M, Handra RJ. Neuroendocrine mechanisms of depression clinical and preclinical evidence. In: Charney DS, Nestler EJ, Sklar P, Buxbaum JD eds. Charney \& Nestler's Neurobiology of Mental Illness. Oxford University Press; 2017.

39. Garcia RG, Zarruk JG, Guzman JC, Barrera C, Pinzon A, Trillos E, et al. Sex differences in cardiac autonomic function of depressed young adults. Biol Psychol 2012;90:179-85.

40. Kemp AH, Quintana DS, Gray MA, Felmingham KL, Brown K, Gatt JM. Impact of depression and antidepressant treatment on heart rate variability: a review and meta-analysis. Biol Psychiatry. 2010;67:1067-74.

41. Stapelberg NJ, Hamilton-Craig I, Neumann DL, Shum DHK, McConnell H. Mind and heart: heart rate variability in major depressive disorder and coronary heart disease - a review and recommendations. Aust N. Z J Psychiatry. 2012;46: 946-57.

42. Chang HA, Chang CC, Tzeng NS, Kuo TB, Lu RB, Huang SY. Cardiac autonomic dysregulation in acute schizophrenia. Acta Neuropsychiatrica. 2013;25:155-64.

43. Smith R, Allen JJB, Thayer JF, Lane RD. Altered functional connectivity between medial prefrontal cortex and the inferior brainstem in major depression during appraisal of subjective emotional responses: a preliminary study. Biol Psychol. 2015;108:13-24.

44. Garcia RGMK, Fluegel B, Holsen L, Aizley H, Remington A, Whitfield-Gabrieli S, et al. Central modulation of parasympathetic response to negative affect is disrupted in major depression: impact of sex. Neuropsychopharmacology. 2015;40: S157-8.

45. Lane RD, Weidenbacher $H$, Smith $R$, Fort $C$, Thayer JF, Allen JJB. Subgenual anterior cingulate cortex activity covariation with cardiac vagal control is altered in depression. J Affect Disord. 2013;150:565-70.

46. Mareckova K, Holsen LM, Admon R, Makris N, Seidman L, Buka S, et al. Brain activity and connectivity in response to negative affective stimuli: impact of dysphoric mood and sex across diagnoses. Hum Brain Mapp. 2016;37:3733-44.

47. Mareckova K, Holsen L, Admon R, Whitfield-Gabrieli S, Seidman LJ, Buka SL, et al. Neural - hormonal responses to negative affective stimuli: impact of dysphoric mood and sex. J Affect Disord. 2017;222:88-97. 
48. Holsen LM, Lee J-H, Spaeth SB, Ogden LA, Klibanski A, Whitfield-Gabrieli S, et al. Brain hypoactivation, autonomic nervous system dysregulation, and gonadal hormones in depression: a preliminary study. Neurosci Lett. 2012;514:57-61.

49. Insel TR, Cuthbert BN, Garvey MA, Heinssen RK, Pine DS, Quinn KJ, et al. Research domain criteria (RDoC): toward a new classification framework for research on mental disorders. Am J Psychiatry. 2010;167:748-51.

50. McNair DM, Droppleman LF, Lorr M. Edits manual for the profile of mood states: POMS. Edits; 1992

51. Spielberger $C$, Gorsuch $R$, Lushene R, Vagg $P$, Jacobs $G$. Manual for the state-trait anxiety inventory. Palo Alto, CA: Consulting Psychologists Press; 1983.

52. CB S. The central autonomic nervous system: conscious visceral perception and autonomic pattern generation. Annu Rev Neurosci. 2002;25:433-69.

53. Task Force of the European Society of Cardiology and the North American Society of Pacing and Electrophysiology. Heart rate variability: standards of measurement, physiological interpretation and clinical use. Circulation. 1996;93:1043-65.

54. Weimer LH. Autonomic testing: common techniques and clinical applications. Neurologist. 2010;16:215-22.

55. Campos LA, Pereira VL, Muralikrishna A, Albarwani S, Brás S, Gouveia S. Mathematical biomarkers for the autonomic regulation of cardiovascular system. Front Physiol. 2013;4:279.

56. Barbieri R, Matten EC, Alabi AA, Brown EN. A point-process model of human heartbeat intervals: new definitions of heart rate and heart rate variability. Am J Physiol Heart Circ Physiol. 2005;288:H424-35.

57. Citi L, Brown EN, Barbieri R. A real-time automated point-process method for the detection and correction of erroneous and ectopic heartbeats. IEEE Trans Biomed Eng. 2012;59:2828-37.

58. Barbieri R, Brown EN. Analysis of heartbeat dynamics by point process adaptive filtering. IEEE Trans Biomed Eng. 2006;53:4-12.

59. Bradley MM, Lang PJ. Measuring emotion: the self-assessment manikin and the semantic differential. J Behav Ther Exp Psychiatry. 1994;25:49-59.

60. Neuroimaging WC. SPM8 Manural. London, UK: Institute of Neuroimaging; 2013.

61. Whitfield-Gabrieli S. REX Software. Cambridge, MA; 2009.

62. McLaren DG, Ries ML, Xu G, Johnson SC. A generalized form of contextdependent psychophysiological interactions (gPPI): a comparison to standard approaches. Neurolmage. 2012;61:1277-86.

63. Maldjian JA, Laurienti PJ, Kraft RA, Burdette JH. An automated method for neuroanatomic and cytoarchitectonic atlas-based interrogation of fMRI data sets. Neurolmage. 2003;19:1233-39.

64. Beissner F, Meissner K, Bär K-J, Napadow V. The autonomic brain: an activation likelihood estimation meta-analysis for central processing of autonomic function. J Neurosci. 2013;33:10503-11.

65. Thayer JF, Ahs F, Fredrikson M, Sollers JJ III, Wager TD. A meta-analysis of heart rate variability and neuroimaging studies: implications for heart rate variability as a marker of stress and health. Neurosci Biobehav Rev. 2012;36:747-56.

66. Benarroch EE. The central autonomic network: functional organization, dysfunction, and perspective. Mayo Clin Proc. 1993;68:988-1001.

67. Critchley HD. Neural mechanisms of autonomic, affective, and cognitive integration. J Comp Neurol. 2005;493:154-66.

68. Davis $M$, Whalen PJ. The amygdala: vigilance and emotion. Mol Psychiatry 2001;6:13-34.
69. Li Q, Zhao Y, Chen Z, Long J, Dai J, Huang X, et al. Meta-analysis of cortical thickness abnormalities in medication-free patients with major depressive disorder. Neuropsychopharmacology. 2020;45:703-12.

70. Burkhouse $\mathrm{KL}$, Jacobs $\mathrm{RH}$, Peters AT, Ajilore O, Watkins ER, Langenecker SA. Neural correlates of rumination in adolescents with remitted major depressive disorder and healthy controls. Cogn, Affect Behav Neurosci. 2017;17: 394-405.

71. Jones EC, Liebel SW, Hallowell ES, Sweet LH. Insula thickness asymmetry relates to risk of major depressive disorder in middle-aged to older adults. Psychiatry Res Neuroimaging. 2019;283:113-17.

72. Sliz D, Hayley S. Major depressive disorder and alterations in insular cortical activity: a review of current functional magnetic imaging research. Front Hum Neurosci. 2012;6:323.

73. Harshaw C. Interoceptive dysfunction: toward an integrated framework for understanding somatic and affective disturbance in depression. Psychological Bull. 2015;141:311-63.

74. Amodio DM, Frith CD. Meeting of minds: the medial frontal cortex and social cognition. Nat Rev Neurosci. 2006;7:268-77.

75. Eden AS, Schreiber J, Anwander A. Emotion regulation and trait anxiety are predicted by the microstructure of fibers between amygdala and prefrontal cortex. J Neurosci. 2015;35:6020-7.

76. De Kloet ER, Vreugdenhil E, Oitzl MS, Joels M. Brain corticosteroid receptor balance in health and disease. Endocr Rev. 1998;19:269-301.

77. Chen L, Wang Y, Niu C, Zhong S, Hu H, Chen $\mathrm{P}$, et al. Common and distinct abnormal frontal-limbic system structural and functional patterns in patients with major depression and bipolar disorder. Neurolmage Clin. 2018;20:42-50.

78. Herman JP, McKlveen JM, Ghosal S, Kopp B, Wulsin A, Makinson R, et al. Regulation of the hypothalamic-pituitary-adrenocortical stress response. Compr Physiol. 2016;6:603-21.

79. Seidman LJ, Faraone SV, Goldstein JM, Kremen WS, Horton NJ, Makris N, et al. Left hippocampal volume as a vulnerability indicator for schizophrenia: a magnetic resonance imaging morphometric study of nonpsychotic first-degree relatives. Arch Gen Psychiatry. 2002;59:839-49.

80. McEwen BS. Stress, adaptation, and disease. Allostasis and allostatic load. Ann N. Y Acad Sci. 1998;840:33-44.

81. Ajayi IE, McGovern AE, Driessen AK, Kerr NF, Mills PC, Mazzone SB. Hippocampal modulation of cardiorespiratory function. Respiratory Physiol Neurobiol. 2018;252-253:18-27.

82. Wei L, Chen H, Wu GR. Structural covariance of the prefrontal-amygdala pathways associated with heart rate variability. Front Hum Neurosci. 2018;12:2.

83. Nugent AC, Bain EE, Thayer JF, Sollers JJ, Drevets WC. Sex differences in the neural correlates of autonomic arousal: a pilot PET study. Int J Psychophysiol. 2011;80:182-91.

84. Goldstein JM, Cherkerzian S, Seidman LJ, Donatelli JA, Remington AG, Tsuang MT, et al. Prenatal maternal immune disruption and sex-dependent risk for psychoses. Psychological Med. 2014;44:3249-61.

85. Garcia R, Gabriel A, Stanford A, Aizley H, Barbieri R, Gitlin D, et al. Effects of respiratory-gated auricular vagal nerve stimulation on central autonomic regulation and mood symptomatology in major depression. Neuropsychopharmacology. 2017;43:S341-2. 